\title{
Bouncing plasmonic waves in half-parabolic potentials
}

\author{
Wei Liu, ${ }^{1,2}$ Dragomir N. Neshev, ${ }^{1}$ Andrey E. Miroshnichenko, ${ }^{1}$ Ilya V. Shadrivov, ${ }^{1}$ and Yuri S. Kivshar ${ }^{1}$ \\ ${ }^{1}$ Nonlinear Physics Centre, Centre for Ultrahigh-bandwidth Devices for Optical Systems (CUDOS), Research School of Physics and \\ Engineering, Australian National University, Canberra, ACT 0200, Australia \\ ${ }^{2}$ College of Optoelectronic Science and Engineering, National University of Defense Technology, Changsha 410073, China
}

(Received 29 September 2011; published 2 December 2011)

\begin{abstract}
We introduce a plasmonic analog for the dynamics of a quantum particle under a linear restoring force bouncing off an impenetrable barrier ("quantum paddle ball"). Paddle-ball-type plasmonic potentials are constructed in quadratically modulated metal-dielectric-metal structures with transverse metallic reflecting walls. We show, both analytically and numerically, the full-wave nature of the phenomenon, including plasmon bouncing and complete wave revivals after interference at the boundary. We show that the plasmon paddle-ball dynamics is effectively wavelength independent, opening opportunities for subwavelength manipulations of polychromatic and ultrashort-pulse plasmons.
\end{abstract}

DOI: 10.1103/PhysRevA.84.063805 PACS number(s): 42.25.Bs, 78.67.-n, 73.20.Mf, 73.40.Rw

\section{INTRODUCTION}

In recent years optics has offered remarkable opportunities to test wave phenomena in various physical systems, including solid-state, relativistic, and nonrelativistic quantum physics $[1,2]$. This is due to the ability to directly visualize the wave function of light beams as well as to build up with unprecedented accuracy the optical potentials, thus engineering the dispersion of waves in the system. Important examples constitute the optical demonstration of Bloch oscillations, dynamic localization, relativistic trembling motion of a free Dirac electron, and quantum bouncing ball [1-3]. The latter originates from the implementation of a half-linear potential model [4], finding its counterparts in cold atoms [5] and neutron physics [6,7]. The linear potentials, however, are anharmonic leading to fractional revivals, phase collapses, and aperiodic oscillations of the bouncing ball $[3,4]$.

Therefore the fundamental harmonic oscillator model of a particle in a parabolic potential has been more widely employed in various fields, including atomic and molecular physics, solid-state physics, and quantum optics $[4,8,9]$. The related half-parabolic potential model, corresponding to a harmonic oscillator with a rigid wall, was also proposed and investigated in terms of a quantum paddle ball $[4,8]$. In a sharp contrast to the anharmonic bouncing balls, paddle balls experience full-wave revivals and periodic beam oscillations with no phase collapses [4]. Also due to its simplicity and harmonicity, the quantum paddle ball can serve as a basic model to explain the full-wave dynamics of matter waves scattered by high potential barriers $[4,10]$, to investigate atomic wave diffraction and interference $[10,11]$, to study the van der Waals force between atom and barrier [12], and to investigate chaotic quantum bouncers [13]. As such the direct optical visualization of the wave dynamics of paddle balls is an attractive milestone.

In this paper, we suggest a plasmonic analog of the quantum paddle ball by employing a half-parabolic potential with a fully repulsive wall (paddle-ball potential). The potential is obtained in a metal-dielectric-metal (MDM) structure with a quadratically modulated dielectric layer and a metallic reflecting wall on one side, as shown in Fig. 1(a). Employing such a potential, we demonstrate both analytically and numerically the wave revivals, oscillations, and interference patterns (in the region close to the reflecting surface) of the plasmonic beam. Furthermore the plasmonic paddle ball can be achieved with broad bandwidth polychromatic light, thus finding possible applications in subwavelength beam steering and manipulations for ultrashort laser pulses.

\section{PLASMONIC PADDLE-BALL POTENTIAL}

The implementation of a plasmonic potential rather than dielectric optical potential carries a number of advantages to our work. The field of plasmonics not only offers a chance to confine light down to the nanoscale [14], but also provides a tool to visualize the wave effects, which are not easy to realize in photonics [15]. This is because in plasmonics one can construct photonic potentials with large absolute values of amplitude and gradient, which are elusive in dielectric structures $[15,16]$.

For a flat MDM slot waveguide with the dielectric layer width of $h$, the effective refractive index (ERI) of the symmetric mode (in terms of the magnetic field distribution) can be expressed as $n_{\text {eff }}(h)=a / h+b$, where $a$ and $b$ are obtained through direct data fitting [16-18]. For example, for $\lambda=0.7 \mu \mathrm{m}, a=(0.0282+0.0002 i) \mu \mathrm{m}$, and $b=1.5874+$ $0.0011 i$; for $\lambda=0.9 \mu \mathrm{m}, a=(0.0264+0.0002 i) \mu \mathrm{m}$, and $b=1.5765+0.0007 i$ when the metal is silver with the permittivity taken from experimental data [19]. In the modulated MDM structure shown in Fig. 1(a) (the thickness of the dielectric layer is $h(x)=h_{0}+x^{2} /\left(2 R_{0}\right)$ for $x \geqslant 0$, with a pure metal wall for $x<0$ ), we obtain a plasmonic paddle-ball potential (graded index distribution) under the condition of $x^{2} \ll 2 h_{0} R_{0}$

$$
n(x) \approx\left\{\begin{array}{l}
\infty(x<0) \\
n_{0}\left(1-\Omega^{2} x^{2} / 2\right)(x \geqslant 0)
\end{array},\right.
$$

where $n_{0}=a / h_{0}+b$, and $\Omega=\sqrt{a /\left(n_{0} R_{0} h_{0}^{2}\right)}=\left[R_{0} h_{0}(1+\right.$ $\left.\left.h_{0} b / a\right)\right]^{-1 / 2}$ is the potential strength. It is worth noting that in Eq. (1) we neglect the field penetration into the left metal wall on the side and the consequent effects, such as phase modification, Goos-Hänchen shift, and bulk mode excitations [20]. The eigenvalues (termed as ERI in the realm of optics) 

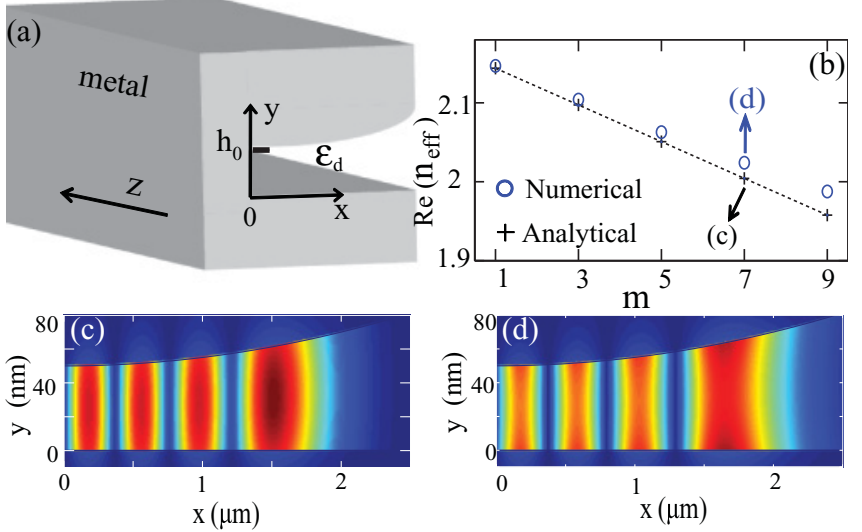

FIG. 1. (Color online) (a) Quadratically modulated MDM structure with a side reflecting metal wall. (b) Real part of the effective refractive index of the modes supported by the structure shown in (a). Both analytical [Eq. (2)] and numerical (mode solver, Lumerical) results are shown. The field distribution of the mode $m=7$ is shown in (c) (analytical, $\left|B_{m}(x, y)\right|$ ) and (d) (numerical, $\left|E_{y}\right|$ ). The metal is silver and other parameters are $h_{0}=50 \mathrm{~nm}, \lambda=0.633 \mu \mathrm{m}$, $R_{0}=100 \mu \mathrm{m}$, and $\varepsilon_{d}=2.25$.

and corresponding eigenmodes of this paddle-ball potential can be easily obtained from its full-potential counterpart $n(x)=n_{0}\left(1-\frac{1}{2} \Omega^{2} x^{2}\right)[x \in(-\infty,+\infty)]$ : Only modes with odd parities of the full potentials can exist in the corresponding paddle-ball potentials, with exactly the same ERI. Those half modes actually provide a complete orthogonal set for the paddle-ball potentials [4]. The eigenvalues of the paddle-ball potential of the structure in Fig. 1(a) are (corresponding to ERI of odd modes in the full parabolic potential [17])

$$
n_{\mathrm{eff}}(m)=n_{0}-\Omega(m+1 / 2) / k(m=1,3,5, \ldots),
$$

where $k=2 \pi / \lambda$ is the wave number in vacuum and the field distributions of the corresponding eigenmodes are: $B_{m}(x, y)=$ $A_{m}(x, y)(x \geqslant 0) ; B_{m}(x, y)=0(x<0)$, where $A_{g}(x, y)(g=$ $0,1,2, \ldots)$ denotes the eigenmodes of the full-potential counterpart in Ref. [17]. In Fig. 1(b) we show the real parts of the ERI and the field distributions of the eigenmodes supported in the structure shown in Fig. 1(a). Both analytical [Eq. (2)] and numerical (Mode solver, Lumerical) results are shown. The dielectric layer between the metal plates is glass with $\varepsilon_{d}=2.25$ and the metal is silver, the permittivity of which is taken from experimental results [19]. Other parameters for Figs. $1(\mathrm{~b})-1(\mathrm{~d})$ are $h_{0}=50 \mathrm{~nm}, \lambda=0.633 \mu \mathrm{m}$, and $R_{0}=$ $100 \mu \mathrm{m}$. Both analytical and numerical results agree very well, except for higher-order modes. This is because higher-order modes spread out more to the larger $x$ region [as shown in Figs. 1(c)-1(d)], where the approximation $x^{2} \ll 2 h_{0} R_{0}$ does not fully hold, and consequently the potential deviates from being exactly parabolic.

\section{PLASMONIC BEAM DYNAMICS IN PADDLE-BALL POTENTIAL}

\section{A. Analytical study of beam evolution}

As the next step, we investigate analytically the beam evolution in the plasmonic potential. The propagation pattern of any incident beam with initial distribution $F(x, y, 0)$ through the waveguide could be expressed as the combination of the eigenmodes of this paddle-ball potential

$$
F(x, y, z)=\sum_{m} \beta_{m} B_{m}(x, y) \exp \left[i k z n_{\mathrm{eff}}(m)\right],
$$

where $\beta_{m}=\iint F(x, y, 0) B_{m}^{*}(x, y) d x d y$ is the expansion coefficient. Meanwhile, considering the special boundary condition, the beam pattern in the half potential could be obtained from the interferences of the incoming wave and its mirror image with respect to the reflecting wall. Hence in our model the expansion for beam propagation could also be expressed as $[4,21]$

$$
F(x, y, z)=\left\{\begin{array}{l}
0(x<0) \\
\psi(x, y, z)-\psi(-x, y, z)(x \geqslant 0)
\end{array} .\right.
$$

Here $\psi(x, y, z)=\sum_{g} \alpha_{g} B_{g}(x, y) \exp \left[i k z n_{\mathrm{eff}}(g)\right]$ and $\alpha_{g}=$ $\iint F(x, y, 0) A_{g}^{*}(x, y) d x d y$ is the corresponding expansion coefficient (expanded into the eigenmodes of the full potential). For the half potential obtained in the structure, as the beam distribution along $y$ direction could be fully characterized by the symmetric mode distribution of the MDM structure [16], we are only interested in the beam propagation pattern in the $x-z$ plane and thus only do the mode expansion along $x$ direction. When the initial beam has a Gaussian distribution along $x$

$$
\bar{F}(x, 0)=\pi^{-\frac{1}{4}} w_{0}^{-\frac{1}{2}} \exp \left[-\left(x-x_{0}\right)^{2} / 2 w_{0}^{2}\right],
$$

where $w_{0}$ characterizes the initial beam width and $x_{0}$ is the beam center offset. According to Eq. (4) and the expression for the beam distribution in full parabolic potential [17], the beam in the $x-z$ domain could be expressed as (under paraxial approximation and ignoring the metallic loss of the plasmonic potential) $[4,21]$

$$
\begin{aligned}
|\bar{F}(x, z)|= & \left(\frac{2}{\xi(z) \sqrt{\pi}}\right)^{1 / 2} \exp \left\{-\frac{1}{2} \frac{x^{2}+x_{0}^{2} \cos ^{2}(\Omega z)}{\xi^{2}(z)}\right\} \\
& \times|P(x, z)-P(-x, z)|,
\end{aligned}
$$

where $P(x, z)=\exp \left[x x_{0} \cos (\Omega z) / \xi^{2}(z)+i x x_{0} \sin (\Omega z) / w_{0}^{2}\right]$ and the dynamic beam width is

$$
\xi(z)=\sqrt{w_{0}^{2} \cos ^{2}(\Omega z)+\eta_{0}^{4} / w_{0}^{2} \sin ^{2}(\Omega z)},
$$

with $\eta_{0}=\left(n_{0} k \Omega\right)^{-1 / 2}$ as the characteristic potential width. Following Eq. (7), the beam experiences periodic oscillations with the period

$$
L=2 \pi / \Omega=2 \pi \sqrt{R_{0} h_{0}\left(1+h_{0} b / a\right)} .
$$

\section{B. Beam-width dependence and numerical results for the beam evolution}

The dynamic beam width is constrained by the potential

$$
\xi_{\max } \xi_{\min }=\eta_{0}^{2}
$$

and the dynamic beam width is constant $\xi(z)=w_{0}$ only if the initial beam width is $w_{0}=\eta_{0}$. When the beam hits the wall, the dynamic beam width is

$$
\xi_{w}=\xi(\Omega z=\pi / 2)=\eta_{0}^{2} / w_{0}
$$




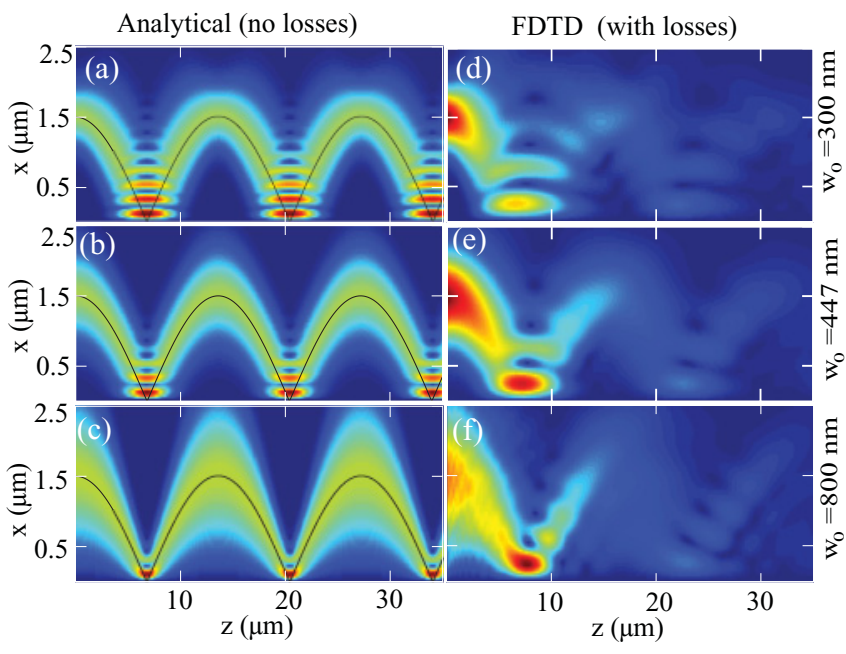

FIG. 2. (Color online) (a)-(c) Analytical results [Eq. (6)] for the beam propagation with three input widths: $w_{0}=300,447$, and $800 \mathrm{~nm}$. Black curves represent the classical trajectories of the beams calculated from Hamiltonian optics. (d)-(f) Numerical finite-difference time-domain (FDTD) results $(|E|)$ on the plane $y=h_{0} / 2=25 \mathrm{~nm}$. Parameters are the same as in Fig. 1 and $x_{0}=1.5 \mu \mathrm{m}$.

which is inversely proportional to $w_{0}$. In Figs. 2(a)-2(c) we show the analytical results of Eq. (6) for three initial beam widths: $w_{0}=300,447$, and $800 \mathrm{~nm}$ when $\lambda=0.633 \mu \mathrm{m}$. The parameters are the same as in Fig. 1 and correspondingly $\eta_{0}=447 \mathrm{~nm}$. The classical trajectories, calculated through Hamiltonian optics [22], are shown by black curves.

Plasmonic paddle balls shown in Figs. 2(a)-2(c) experience full beam revivals and periodic beam oscillations. This is due to the harmonic nature of the paddle-ball potentials, in other words, the symmetry of the Hamiltonian in phase space and thus the equidistant energy levels (or equidistant ERIs of eigenmodes in optics) [4,23]. Meanwhile, similar to photonic bouncing balls, plasmonic paddle ball shows significant interference patterns in the region close to the walls, which come from the interference of the incoming and reflecting beams. It is worth mentioning that smaller $w_{0}$ would lead to larger dynamic beam width $\xi_{w}[\mathrm{Eq} .(10)]$ and thus larger overlapping region of the incoming and reflecting beams. This would lead to larger interference regions and thus more minimum lobes [Figs. 2(a)-2(c)].

In Figs. 2(d)-2(e) we show correspondingly the FDTD results for $|E|$ on the plane $y=h_{0} / 2=25 \mathrm{~nm}$ (Lumerical), with the Ohmic losses of metal included. The discrepancies between the analytical and the numerical results come from both metallic losses and the paddle-ball potential approximation [Eq. (1)].

\section{Spectral dependence of beam evolution}

Finally, we study the dispersion of the plasmonic paddleball potentials. The period of the beam oscillations $L$ depends on the potential strength $\Omega$. According to Eq. (8), the period of the oscillation depends on $b / a$ only. In Fig. 3(a) we plot the real part of $b / a$ in the wavelength range of $0.5-0.9 \mu \mathrm{m}$. Considering that this ratio changes slowly with wavelength, we
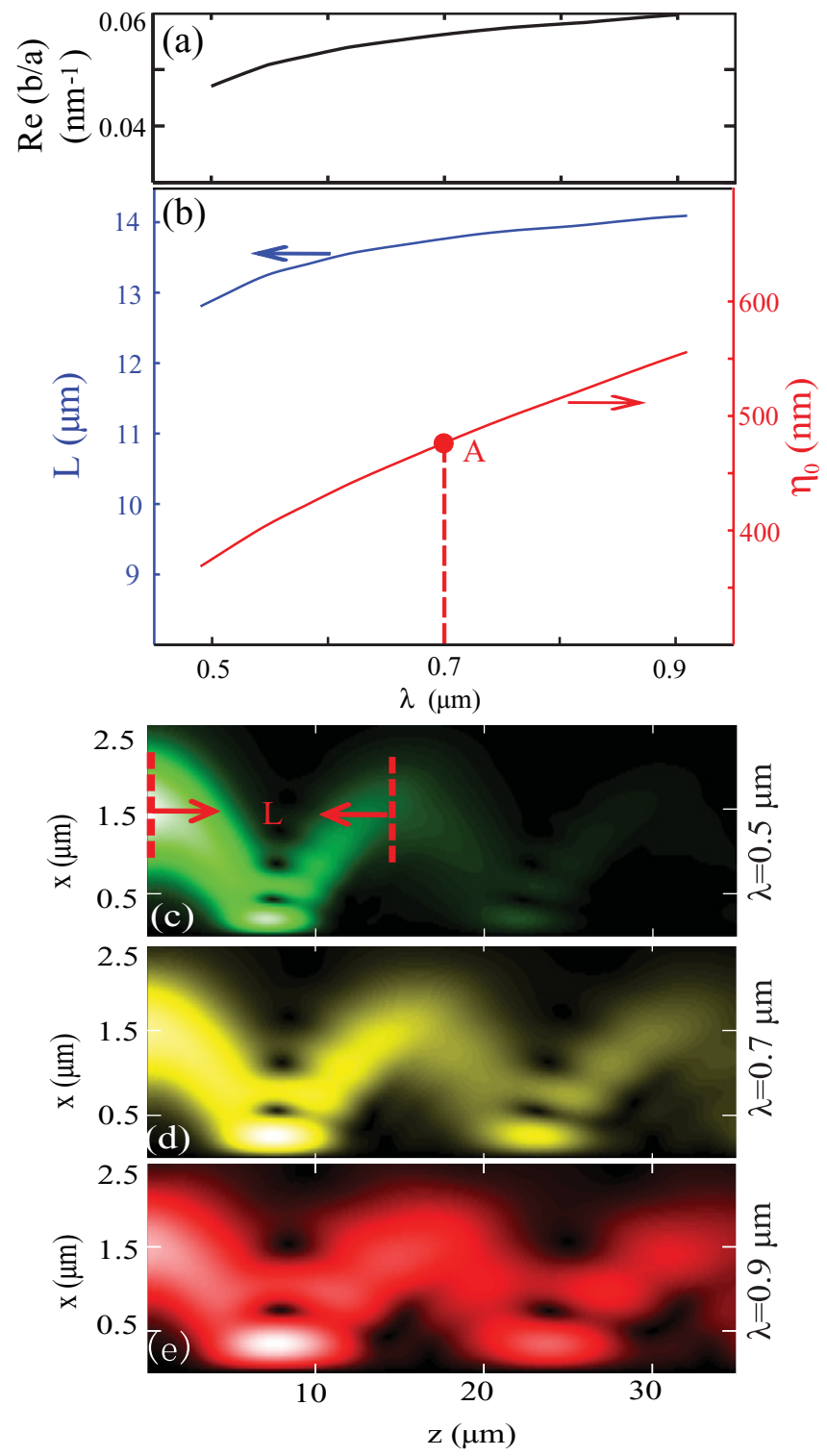

FIG. 3. (Color online) (a) Real part of fitting parameter $b / a$ and (b) characteristic potential width $\eta_{0}$ and oscillation period $L$ for the wavelength range of $0.5-0.9 \mu \mathrm{m}$. (c)-(e) FDTD results $(|E|)$ for beam propagation on the plane $y=h_{0} / 2=25 \mathrm{~nm}$ for three wavelengths of $\lambda=0.5,0.7$, and $0.9 \mu \mathrm{m}$. For all the three cases, $w_{0}$ is fixed at $482 \mathrm{~nm}$, which is the characteristic potential width of $\lambda=0.7 \mu \mathrm{m}$, as indicated by point A in (b). Except for the wavelength, other parameters are the same as in Fig. 1 and $x_{0}=1.5 \mu \mathrm{m}$.

expect multicolor plasmonic paddle balls with almost the same oscillating period: polychromatic plasmonic paddle balls. In Fig. 3(b) we show both the characteristic potential width $\eta_{0}$ and oscillation period $L$ for the wavelength range of $0.5-0.9 \mu \mathrm{m}$. Other parameters are the same as in Fig. 1. It is obvious that for this wavelength range of more than an optical octave, $L$ is almost the same and the aberration is within $1 \mu \mathrm{m}$.

Then we show the beam distributions on the plane $y=$ $h_{0} / 2=25 \mathrm{~nm}$ for three wavelengths of $\lambda=0.5,0.7$, and $0.9 \mu \mathrm{m}$ in Figs. 3(c)-3(e) respectively (FDTD, $|E|$ ). The initial beam width $w_{0}$ is fixed at $476 \mathrm{~nm}$, which is the characteristic potential width for $\lambda=0.7 \mu \mathrm{m}$ [point A in Fig. 3(b)]. As is 
shown, the plasmonic analog of a paddle ball of different colors exhibits almost dispersionless oscillation period, justifying the claim of polychromatic behavior of the plasmonic analog of a paddle ball. Meanwhile $\eta_{0}$ is of significant differences for beams of different wavelengths [Fig. 3(b)]. This means that with fixed $w_{0}$, the dynamic beam width $\xi_{w}$ changes with wavelength [Eq. (10)], leading to different interference pictures for light of different wavelengths.

\section{CONCLUSION AND PERSPECTIVES}

In summary, we have introduced a plasmonic analog of a quantum paddle ball through employing a quadratically modulated MDM structure with a reflecting wall on the side. We have described beam revivals, periodic beam oscillations, and significant interference patterns close to the wall, which resemble the full-wave dynamics of the corresponding propagating matter waves. We have also demonstrated that the plasmonic paddle ball operates in a polychromatic regime, which may find applications in broadband subwavelength beam manipulations, polychromatic plasmon focusing, and ultrashort-pulse plasmonic nanolasers.

The structure proposed in this paper is quite practical considering that it corresponds to a broad metal wire (with diameter of several hundred microns) above a metal plate with another reflecting metal wall on the side. Also when reflecting walls are introduced at the potential bottom, the plasmonic paddle-ball potential could be realized in air-polymer-metal structures, which could be characterized directly by fluorescence imaging or leakage radiation microscopy [15,24]. It is worth mentioning that paddle-ball potential is not confined to the field of plasmonics and could be easily extended to other branches of nanophotonics and graded index structures.

\section{ACKNOWLEDGMENTS}

We acknowledge the support of the Australian Research Council Centre of Excellence for Ultrahigh Bandwidth Devices for Optical Systems (Project No. CE110001018) and the National Computing Infrastructure Merit Allocation Scheme.
[1] S. Longhi, Laser Photonics Rev. 3, 243 (2009).

[2] F. Dreisow, M. Heinrich, R. Keil, A. Tunnermann, S. Nolte, S. Longhi, and A. Szameit, Phys. Rev. Lett. 105, 143902 (2010).

[3] G. Della Valle, M. Savoini, M. Ornigotti, P. Laporta, V. Foglietti, M. Finazzi, L. Duo, and S. Longhi, Phys. Rev. Lett. 102, 180402 (2009).

[4] R. W. Robinett, Phys. Rep. 392, 1 (2004).

[5] C. G. Aminoff, A. M. Steane, P. Bouyer, P. Desbiolles, J. Dalibard, and C. Cohen-Tannoudji, Phys. Rev. Lett. 71, 3083 (1993).

[6] V. V. Nesvizhevsky, H. G. Borner, A. K. Petukhov, H. Abele, S. Baeszler, F. J. Ruesz, T. Stoferle, A. Westphal, A. M. Gagarski, G. A. Petrov, and A. V. Strelkov, Nature (London) 415, 297 (2002).

[7] V. V. Nesvizhevsky, A. Y. Voronin, R. Cubitt, and K. V. Protasov, Nature Phys. 6, 114 (2010).

[8] R. W. Robinett, Quantum Mechanics (Oxford University Press, New York, 1997).

[9] R. J. Glauber, Phys. Rev. 130, 2529 (1963).

[10] J. P. Dowling and J. Gea-Banacloche, Adv. Atom. Mol. Opt. Phys. 37, 1 (1996).

[11] P. Szriftgiser, D. Guéry-Odelin, M. Arndt, and J. Dalibard, Phys. Rev. Lett. 77, 4 (1996).
[12] A. Landragin, J. Y. Courtois, G. Labeyrie, N. Vansteenkiste, C. I. Westbrook, and A. Aspect, Phys. Rev. Lett. 77, 1464 (1996).

[13] S. T. Dembinski, A. J. Makowski, and P. Peplowski, Phys. Rev. Lett. 70, 1093 (1993).

[14] D. K. Gramotnev and S. I. Bozhevolnyi, Nature Photonics 4, 83 (2010).

[15] T. Zentgraf, Y. Liu, M. H. Mikkelsen, J. Valentine, and X. Zhang, Nature Nano. 6, 151 (2011).

[16] S. I. Bozhevolnyi and K. V. Nerkararyan, Opt. Express 17, 10327 (2009).

[17] W. Liu, D. N. Neshev, A. E. Miroshnichenko, I. V. Shadrivov, and Y. S. Kivshar, Phys. Rev. B 83, 073404 (2011).

[18] W. Liu, D. N. Neshev, I. V. Shadrivov, A. E. Miroshnichenko, and Y. S. Kivshar, Opt. Lett. 36, 1164 (2011).

[19] E. D. Palik and G. Ghosh, Handbook of Optical Constants of Solids (Academic Press, San Diego, 1998).

[20] A. A. Maradudin, R. F. Wallis, and G. I. Stegeman, Prog. Surf. Sci. 33, 171 (1990)

[21] M. Andrews, Am. J. Phys. 66, 252 (1998).

[22] J. A. Arnaud, Beam and Fiber Optics (Academic Press, New York, 1976).

[23] A. Royer, Am. J. Phys. 64, 1393 (1996).

[24] G. Della Valle and S. Longhi, Phys. Rev. B 82, 153411 (2010). 